\title{
Semiclassical variational calculation of liquid-drop model coefficients for metal clusters ${ }^{\star}$
}

\author{
M. Seidl, M.E. Spina, and M. Brack \\ Institut für Theoretische Physik, Universität Regensburg, W-8400 Regensburg, Federal Republic of Germany
}

Received 10 September 1990

\begin{abstract}
We report on semiclassical density variational calculations for spherical alkali metal clusters in the jellium model. We derive liquid-drop model expansions for total energy, ionisation potential and electron affinity and test the coefficients numerically for clusters with up to $N=10^{5}$ atoms. From the limit $N \rightarrow \infty$, we obtain excellent agreement with surface tensions and work functions evaluated for an infinite plane metal surface.
\end{abstract}

PACS: $36.40 .+$ d; 31.20.Lr; 31.20.Sy; 03.65.Sq

We have extended recent semiclassical density variational calculations [1] for spherical alkali metal clusters in the jellium model, where the positive ionic charge distribution is replaced by a homogeneous background density $\rho_{I 0}$ inside a sphere of radius $R_{I}=r_{s} N^{1 / 3}$, where $r_{s}$ $=\left[4 \pi \rho_{I 0} / 3\right]^{-1 / 3}$ is the Wigner-Seitz radius and $N$ the number of atoms. The valence electrons are treated in density functional theory, writing the total energy of the system as a functional $E[\rho]=T_{s}[\rho]+E_{C}[\rho]+E_{x c}[\rho]$ of the electronic ground-state density $\rho(r)$. For the (noninteracting) kinetic energy $T_{s}[\rho]$ we use the full fourthorder extended Thomas-Fermi (ETF) functional [2], $\mathrm{E}_{c}[\rho]$ is the electrostatic Coulomb energy (including that of the ions), and the exchange-correlation energy $E_{x c}[\rho]$ is taken in the local density approximation of Gunnarsson and Lundqvist [3]. The ground-state density of the system is determined by minimizing the energy $E[\rho]$. We choose a variational approach to this problem, using a parametrized form of the electron density

$$
\rho(r)=\frac{\rho_{0}}{\left[1+\exp \left(\frac{r-R}{a}\right)\right]^{\gamma}}, \quad \int \rho(r) \mathrm{d}^{3} r=N+z
$$

\footnotetext{
* Work partially supported by Deutsche Forschungsgemeinschaft
}

and minimizing the energy with respect to the three parameters $\rho_{0}, a$ and $\gamma . R$ is used for normalization to the number $z$ of excess electrons in a cluster with $N$ atoms.

Due to the semiclassical nature of the ETF kinetic energy functional and the form (1) of our variational densities, we cannot account for shell effects which are due to the quantal nature of the electronic single-particle states (see, e.g., [4]). We can, however, obtain a selfconsistent description of average static properties of alkali clusters. Indeed, our results reproduce very well on the average the microscopic Kohn-Sham results for spherical clusters $[4,1]$, as well as for metal surfaces $[5,6]$ (using the limit $N \rightarrow \infty$ ).

Our approach is particularly well suited for studying the asymptotic behaviour of cluster properties in the limit $N \rightarrow \infty$. Our present aim is to investigate this limit, both analytically and numerically, for the total ground-state energy $E(N, z)$ and two quantities derived from it: the ionisation potential $I$ and the electron affinity $A$

$$
\begin{aligned}
& I(N)=E(N,-1)-E(N, 0), \\
& A(N)=E(N, 0)-E(N,+1) .
\end{aligned}
$$

In order to study the asymptotic behaviour of these quantities, we start from a leptodermous expansion of the energy $E(N, z)$ in powers of the small quantity $a / R$, which leads to a liquid-drop model type expansion in powers of $N^{-1 / 3}$. (See, e.g., [7] for a similar expansion of the total binding energy of atomic nuclei). This is very difficult to perform analytically with the density profile (1). However, for simple analytic profiles (e.g., a symmetric doubleexponential or trapezoidal form) we arrive at the following result for the leading terms:

$$
\begin{gathered}
E(N, z)=E_{C}^{c l}(N, z)-z \Delta \varphi^{\text {out }}+e_{b}(N+z) \\
+\left(\text { terms } \propto N^{2 / 3}\right)+\ldots
\end{gathered}
$$

Hereby $E_{C}^{c l}$ is the classical Coulomb energy corresponding to a constant sharp-edged electron density

$E_{C}^{c l}(N, z)=\frac{3}{5} \frac{e^{2}}{r_{s}}\left(\frac{3}{2}(N+z)^{5 / 3}+N^{5 / 3}-\frac{5}{2}(N+z) N^{2 / 3}\right)$. 
In (3), $\Delta \varphi^{\text {out }}$ is the outer part of the Coulomb barrier of an infinite plane metal surface, i.e., its electrostatic potential $\varphi$ taken between an infinite distance outside the metal and the jellium edge:

$\Delta \varphi^{\text {out }}=\varphi(\infty)-\varphi(0)=4 \pi e^{2} \int_{0}^{\infty} u \delta \rho(u) \mathrm{d} u$,

where $\delta \rho(u)=\rho(u)-\rho_{I 0} \Theta(-u)$ and $u$ is the distance from the jellium edge along the normal to the surface. Finally, $e_{b}$ in (3) is the bulk energy per electron. For the more realistic electronic densities (1), we have verified (3) numerically, calculating $\Delta \varphi^{\text {out }}$ in (5) with the asymptotic profiles $\rho(r)$ (i.e., extrapolating their parameters to $N^{-1 / 3} \rightarrow 0$, replacing $r$ by $u$ and adjusting $R$ to impose overall charge neutrality).

From $(3,4)$ one finds the following asymptotic expressions for the quantities $I$ and $A$ :

$$
\begin{aligned}
& I=W+\alpha \frac{e^{2}}{R_{I}}+O\left(R_{I}^{-2}\right), \\
& A=W-\beta \frac{e^{2}}{R_{I}}+O\left(R_{I}^{-2}\right) .
\end{aligned}
$$

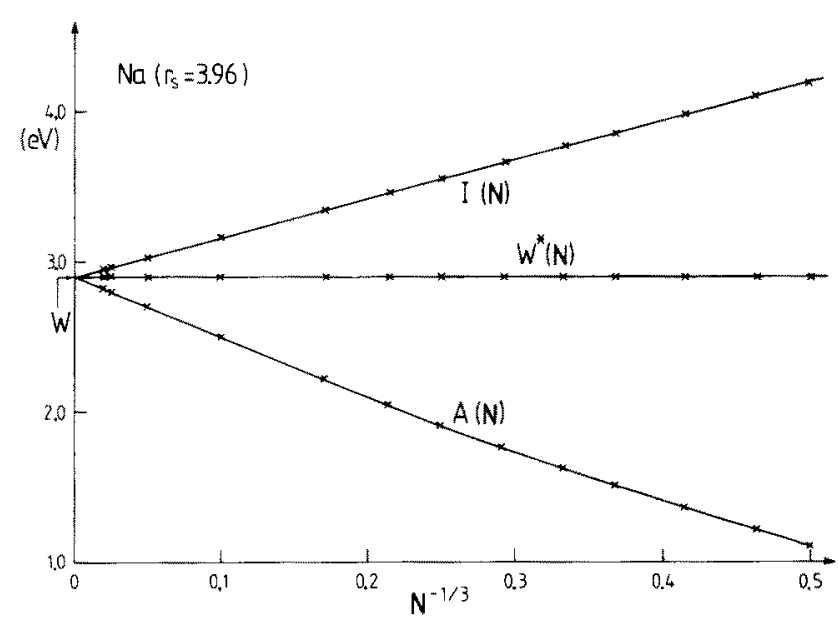

Fig. 1. Ionisation potential $I$, electron affinity $A$ and work function $W^{*}$ (see text for definition) of spherical $\mathrm{Na}$ clusters with $8 \leq N \leq 125000$, plotted versus $N^{-1 / 3}$. Shown on the left is the theoretical bulk work function $W, \mathrm{Eq} .(7)$
They both have the same $N \rightarrow \infty$ limit, namely the bulk work function $W$ which from the above is found to be

$W=\Delta \varphi^{\text {out }}-e_{b}$,

in agreement with an older analytical result [8].

Figure 1 shows some typical results of $I$ and $A$ for $\mathrm{Na}$ clusters $\left(r_{s}=3.96\right.$ a.u. $)$ with $8 \leq N \leq 125000$, plotted versus $N^{-1 / 3} \propto 1 / R_{I}$. Note that $I$ is almost linear in $N^{-1 / 3}$, whereas $A$ has a clear curvature due to the terms $O\left(R_{I}^{-2}\right)$ in (6). In a recent detailed analysis [9] of experimental ionisation potentials and electron affinities, we find that this curvature can, indeed, be observed as an average trend and is very well reproduced by our ETF variational results, even down to the smallest clusters. The quantity $W^{*}$ also shown in Fig. 1 is defined like $W$ through $(5,7)$, but in terms of the variational density $\rho(r)$ of the finite cluster with $N$ atoms. Its constancy, even down to $N=8$, and the perfect agreement with the asymptotic value $W$ are quite remarkable

By least-squares fit to $I(N)$ and $A(N)$ for $1000 \leq$ $N \leq 150000$, we have determined the parameters $W, \alpha$ and $\beta$ in the asymptotic expressions (6); they are given in Table $1 . W$ is found to agree with the theoretical bulk value (7) to within four decimals. Note that the slope parameters $\alpha$ and $\beta$ have a slow, but systematic dependence on $r_{s}$, i.e. on the electron density. Their values (at least for $r_{s} \geq 4$ a.u.) are close to the values $\frac{3}{8}$ and $\frac{5}{8}$, respectively, used by many authors who refer to classical image charge arguments. We consider this to be an accident. In fact, the contribution of the classical Coulomb energy in (4) alone would lead to $\alpha=\beta=\frac{1}{2}$ (see also [10]). The differences from the value $\frac{1}{2}$ are due to the kinetic, exchange and correlation energies and the fact that the electron density has a quantum-mechanical tail (spill-out). Note, however, that the sum of $\alpha$ and $\beta$ is close to unity for all values of $r_{s}$, in agreement with some theoretical arguments [11].

The total energy $E(N)=E(N, 0)$ of a neutral cluster has from the above the following liquid-drop model expansion

$E(N)=e_{b} N+a_{s} N^{2 / 3}+a_{c} N^{1 / 3}+\ldots$

Table 1 contains also the values of the surface energy $a_{s}$ and curvature energy $a_{c}$ for various $r_{s}$, determined by least-squares fit of our numerical variational energies to (8) for clusters with $N$ up to $10^{5}$. From $a_{s}$ we get the surface tension $\sigma$ using the relation $a_{s}=4 \pi r_{s}^{2} \sigma$. Our

Table 1. Liquid-drop expansion coefficients, obtained by least-squares fit, for various values of $r_{s}$. (Some of the values differ slightly from those given in Table II of Ref. 6 due to an improved numerical integration used here)

\begin{tabular}{llllllll}
\hline $\begin{array}{l}r_{s} \\
\text { (a.u.) }\end{array}$ & $\alpha$ & $\beta$ & \multicolumn{1}{l}{$\begin{array}{l}a_{s} \\
\text { (Ry) }\end{array}$} & $\begin{array}{l}a_{c} \\
(\mathrm{Ry})\end{array}$ & $\begin{array}{l}\sigma \\
\left(\mathrm{erg} \mathrm{cm}^{-2}\right)\end{array}$ & $\begin{array}{l}\sigma_{\infty} \\
\left(\mathrm{erg} \mathrm{cm}^{-2}\right)\end{array}$ & $\begin{array}{l}W \\
(\mathrm{eV})\end{array}$ \\
\hline 2.0 & 0.423 & 0.569 & -0.05492 & 0.04632 & -850.0 & -850.0 & 3.612 \\
3.0 & 0.410 & 0.587 & 0.03052 & 0.02357 & 209.9 & 210.5 & 3.252 \\
4.0 & 0.398 & 0.601 & 0.03994 & 0.01123 & 154.5 & 154.9 & 2.885 \\
5.0 & 0.387 & 0.612 & 0.03783 & 0.00380 & 93.69 & 93.92 & 2.569 \\
6.0 & 0.380 & 0.619 & 0.03392 & -0.00083 & 58.33 & 58.48 & 2.303 \\
\hline
\end{tabular}


values $\sigma$ agree very well with the results $\sigma_{\infty}$ (both shown in the table) of independent variational calculations for an infinite plane metal surface using the same density profiles (1) replacing, again, $r$ by $u$ as in (5). (The unrealistic values of $\sigma$ for $r_{s} \leqslant 3$ a.u. are a well-known break down of the jellium model [5].)

A good agreement of surface energies $\sigma$ and work functions $W$ is also found [6] when comparing our restricted variational method with an exact numerical solution of the Euler equation, obtained recently by Tarazona and Chacón [12]; using identical energy density functionals (fourth-order ETF kinetic energy and Wigner's exchange-correlation energy [13]). This shows that our parametrisation (1) of the electronic density is, indeed, flexible enough for a satisfactory determination of the static average properties (at least of alkali clusters) discussed here. Note that in all clusters $\gamma \neq 1$, leading to an asymmetric surface profile and a density tail which is very close to that of microscopic quantum-mechanical calculations [4].

\section{References}

1. Brack, M.: Phys. Rev. B 39, 3533 (1989)

2. Hodges, C.H.: Can. J. Phys. 51, 1428 (1973)

3. Gunnarsson, O., Lundqvist, B.I.: Phys. Rev. B 13, 4274 (1976)

4. Ekardt, W.: Phys. Rev. B 29, 1558 (1984); Beck, D.E.: Phys. Rev. B 30, 6935 (1984)

5. Lang, N.D., Kohn, W.: Phys. Rev. B 1, 4555 (1970); B 3, 1215 (1971)

6. Spina, M.E., Seidl, M., Brack, M.: Symposium on Atomic and Surface Physics 'SASP90'. Märk, T.D., Howorka, F. (eds.), p. 426. Innsbruck University 1990

7. Brack, M., Guet, C., Håkansson, H.-B.: Phys. Rep. 123, 275 (1985)

8. Mahan, G.D., Schaich, W.L.: Phys. Rev. B 10, 2647 (1974)

9. Seidl, M., Meiwes-Broer, K.-H., Brack, M.: Phys. Rev. B (submitted)

10. Makov, G., Nitzan, A., Brus, L.E.: J. Chem. Phys. 88, 5076 (1988); Perdew, J.P.: In: Condensed matter theories. Keller, J. (ed.), Vol. 4, p. 149. New York: Plenum 1989

11. Engel, E., Perdew, J.P.: Phys. Rev. B (in press)

12. Tarazona, P., Chacón, E.: Phys. Rev. B 39, 10366 (1989)

13. Wigner, E.P.: Phys. Rev. 46, 1002 (1934) 